\title{
Rastreio e associações ao câncer cervical
}

\section{Screening and associations to cervical cancer}

\section{Seguimiento y asociaciones al cáncer de cuello uterino}

\author{
Amine Selim de Salles Gonçalves Melado ${ }^{1}$, Icaro Borges de Oliveira ${ }^{\circledR}$, Filipe Alvarenga Caetano Vitorino ${ }^{\circledR}$, Janinne Fachetti \\ Rocha $^{\circledR}$, Gustavo Enrico Cabral Ruschi ${ }^{\circledR}$, Waleska Souza Reisman ${ }^{\circledR}$, Ana Rosa Murad Szpilman ${ }^{1}{ }^{\circledR}$ \\ ${ }^{1}$ Universidade Vila Velha (UVV), Vila Velha/ES
}

\section{Resumo}

Introdução: De acordo com o Instituto Nacional do Câncer (INCA) a estimativa de novos casos de câncer do colo do útero (CCU) para o Estado do Espírito Santo, no triênio 2020-2022, é de 11,65 por 100.000 habitantes. Objetivos: Traçar o perfil clínico-epidemiológico das usuárias do serviço de atenção integral à saúde da mulher da policlínica da Universidade Vila Velha (UVV), a partir dos determinantes da consulta ginecológica, e correlacionar aos achados citológicos e histológicos. Métodos: Estudo de corte transversal, observacional e retrospectivo envolvendo 590 prontuários de pacientes, atendidas entre fevereiro de 2011 e julho de 2016 no serviço de atenção integral à saúde da mulher na policlínica/UVV. Resultados: A média de idade das mulheres incluídas nesse estudo foi de 39,5 anos $(\mathrm{DP} \pm 16,2)$. Os fatores de risco como estado civil e tempo de relacionamento conjugal, multiparidade, e uso de contraceptivos hormonais orais foram associados a maior chance de CCU. Associação estatística da variável idade não evidenciou risco de CCU. Conclusões: Os resultados vão de acordo com os preconizados pelo INCA e Ministério da Saúde. Isso reforça a importância da rastreabilidade na atenção primária e os fatores que interferem na mesma.

Palavras-chave: Ginecologia; Displasia do Colo do Útero; Assistência Integral à Saúde; Risco Moral no Setor de Saúde Suplementar; Administração em Saúde Pública.

Como citar: Melado ASSG, Oliveira IB, Vitorino FAC, Rocha JF, Ruschi GEC, Reisman WS, Szpilman ARM. Rastreio e associações ao câncer cervical. Rev Bras Med Fam Comunidade. 2021;16(43):2992. https://doi.org/10.5712/rbmfc16(43)2992

\author{
Autor correspondente: \\ Ana Rosa Murad Szpilman. \\ E-mail: szpanarm @gmail.com \\ Fonte de financiamento: \\ não se aplica. \\ Parecer CEP: \\ 2.679.489 \\ Procedência: \\ não encomendado. \\ Avaliação por pares: \\ externa. \\ Recebido em: 16/03/2021. \\ Aprovado em: 28/05/2021.
}




\begin{abstract}
Introduction: According to the National Cancer Institute (INCA), the estimate of new cases of cervical cancer (CC) for the State of Espírito Santo, in the 2020-2022 period, is 11.65 per 100,000 inhabitants. Objectives: To trace the clinical-epidemiological profile of users of the comprehensive women's health care service at the University Vila Velha (UVV), based on the determinants of gynecological consultation, and to correlate with cytological and histological findings. Methods: Cross-sectional, observational and retrospective study involving 590 medical records of patients, attended between February 2011 and July 2016 at the comprehensive care service for women's health in the polyclinic/UVV. Results: The average age of women included in this study was 39.5 years $(S D \pm 16.2)$. Risk factors such as marital status and length of marital relationship, multiparity, and use of oral hormonal contraceptives were associated with an increased chance of CC. Statistical association of the age variable did not show a risk of CC. Conclusion: The results are in line with those recommended by INCA and the Ministry of Health. This reinforces the importance of traceability in primary care and the interfering factors.
\end{abstract}

Keywords: Gynecology; Uterine Cervical Dysplasia; Comprehensive Health Care; Moral Risk in Supplementary Health Insurance; Public Health Administration.

\title{
Resumen
}

Introducción: Según el Instituto Nacional del Cáncer (INCA), la estimación de nuevos casos de cáncer de cuello uterino (CC) para el Estado de Espírito Santo en el período 2020-2022 es de 11,65 por 100.000 habitantes. Objetivo: Trazar el perfil clínico-epidemiológico de las usuarias del servicio de atención integral de salud a la mujer de la policlínica Universitaria Vila Velha (UVV), a partir de los determinantes de la consulta ginecológica, y correlacionarlo con hallazgos citológicos e histológicos. Métodos: Estudio transversal, observacional y retrospectivo que involucró a 590 historias clínicas de pacientes, atendidas entre febrero de 2011 y julio de 2016 en el servicio de atención integral de salud de la mujer de la policlínica/UVV. Resultados: La edad promedia de las mujeres incluidas en este estudio fue de 39,5 años (DE $\pm 16,2$ ). Los factores de riesgo como el estado civil y la duración de la relación matrimonial, la multiparidad y el uso de anticonceptivos hormonales orales se asociaron con una mayor probabilidad de CC. La asociación estadística de la variable edad no mostró riesgo de CC. Conclusiones: Los resultados están en línea con los recomendados por INCA y el Ministerio de Salud, lo que refuerza la importancia de la trazabilidad en la atención primaria y los factores que interfieren en ella.

Palabras clave: Ginecología; Displasia del Cuello del Útero; Atención Integral de Salud; Riesgo Moral en los Seguros de Salud; Administración en Salud Pública.

\section{INTRODUÇÃO}

O câncer de colo de útero (CCU), em diversas partes do mundo, continua sendo um sério problema de saúde pública, a despeito dos avanços observados no tripé diagnóstico clássico (citopatológico, colposcopia e biópsia) nas últimas décadas, com a inserção da biologia molecular em diversos protocolos de rastreamento. No Brasil, o CCU representa o $3^{\circ}$ câncer mais comum entre as brasileiras de 15 a 44 anos. ${ }^{1}$

De acordo com o Instituto Nacional do Câncer, ${ }^{2}$ o número de novos casos de CCU esperados para o Brasil, para cada ano do triênio 2020-2022, é de 16.590, com um risco estimado de 15,43 casos a cada 100 mil mulheres. Já para o Estado do Espírito Santo, estima-se 240 novos casos, o que representa uma taxa de incidência de 11,65 por 100.000 habitantes.

O principal fator associado à ocorrência de CCU é a infecção pelo papilomavírus humano (HPV), o qual desempenha um papel crucial na história natural dessa doença. ${ }^{3}$

No Brasil, estudos registraram um perfil de prevalência da infecção por HPV de alto risco semelhante ao dos países subdesenvolvidos: $17,8 \%$ a $27 \%$. Esse quadro também se reflete nas taxas de morbidade e mortalidade, uma vez que as medidas de combate à doença dependem de programas públicos de prevenção e controle da doença. ${ }^{4}$ Tão importante quanto o tratamento efetivo do CCU é a detecção precoce da doença. Países que atingem cobertura do exame citopatológico superior a 70\%, apresentam taxas inferiores ou iguais a duas mortes por 100 mil mulheres por ano. ${ }^{5}$

O INCA ${ }^{6}$ estabelece que o exame citopatológico, empregado no rastreio de CCU, deve ser realizado em brasileiras de 25 a 64 anos de idade que já tenham vida sexualmente ativa, uma vez por ano e, após dois 
exames anuais consecutivos com resultados negativos, a cada três anos. O diagnóstico precoce e o êxito no rastreamento do CCU e de suas lesões precursoras dependerão, além de outros fatores, da acuidade e precisão em diagnosticar corretamente lesões neoplásicas e pré-neoplásicas verdadeiras, daqueles casos que não apresentam qualquer tipo de alteração epitelial. ${ }^{7}$

Nesse contexto, o serviço de atenção integral à saúde da mulher - policlínica de referência da Universidade Vila Velha (UVV), oferece à população feminina da região da grande Vitória/ES, serviços de assistência à saúde da mulher bem como de prevenção do CCU. Assim, esse estudo buscou identificar o perfil clínico epidemiológico dessas pacientes, e definir a prevalência das alterações citológicas e histológicas das mulheres submetidas à rotina de rastreamento do CCU, no período de fevereiro de 2011 a julho de 2016, evidenciando os fatores que se associam às lesões no colo do útero e suas causas atribuídas. Dessa forma poderemos observar o papel da Saúde Suplementar na prevenção do câncer de colo uterino.

\section{MÉTODOS}

Consiste em um estudo observacional descritivo transversal, realizado por meio de consulta aos dados disponíveis em 590 prontuários das pacientes atendidas no serviço de atenção integral à saúde da mulher da policlínica da UVV, no período de fevereiro de 2011 a julho de 2016.

Todos os prontuários analisados abrangeram consultas com anamnese, exame físico geral e ginecológico, com coleta citopatológica, sendo as mulheres encaminhadas para a colposcopia e biópsia de acordo com o protocolo de rastreamento do câncer de colo de útero do Instituto Nacional do Câncer (INCA/MS).

A coleta de dados foi realizada a partir dos registros dos prontuários analisados e correlacionados aos dados sociodemográficos (idade, estado civil, raça/cor); antecedentes ginecológicos (menarca, início da atividade sexual, realização do último exame citopatológico); antecedentes obstétricos (número de gestações, número de partos e abortos) e dados referentes ao rastreamento do CCU (citopatológico e biópsia).

Os dados foram registrados em meio eletrônico e analisados estatisticamente por meio da construção de tabelas e gráficos e do cálculo de proporções, de médias e respectivos desvios padrões, bem como do $p$-valor para os testes inferenciais que foram aplicados. Adotou-se nível de significância de $5 \%$.

O comitê de ética em pesquisa com seres humanos da Universidade Vila Velha, aprovou a pesquisa sob número 2.679.489, em 28 de maio de 2018.

\section{RESULTADOS}

O perfil sociodemográfico e comportamental das pacientes atendidas no serviço de atenção integral à saúde da mulher da policlínica da UVV, no período de janeiro de 2011 a julho de 2016, foi obtido por meio da análise de 590 prontuários. Mulheres entre 26 e 45 anos totalizaram 40,1\%, 72,6\% se autodefiniram como pardas e 55,1\% como solteira/separada/viúva (Gráfico 1). A rotina ginecológica foi o principal motivo de consulta de 433 pacientes, e dentre esse grupo, a realização do exame citopatológico foi determinante para 31,2\% delas.

A idade média em que ocorreu a menarca foi aos 12,7 anos. Os registros médios de gestações contabilizaram 1,98; de partos 1,62; e de abortos 0,34. Das 255 pacientes em uso de métodos contraceptivos, $59,2 \%$ adotaram contraceptivo hormonal $(\mathrm{ACH}), 38,2 \%$ não fazem uso de qualquer método contraceptivo e apenas $14,1 \%$ utilizam preservativo de barreira masculino (Gráfico 2 ). 
Gráfico 1. Perfil sociodemográfico e comportamental das mulheres atendidas na policlínica de referência da UVV, no período de 2011 a 2016.

\section{Perfil sociodemográfico e comportamental}

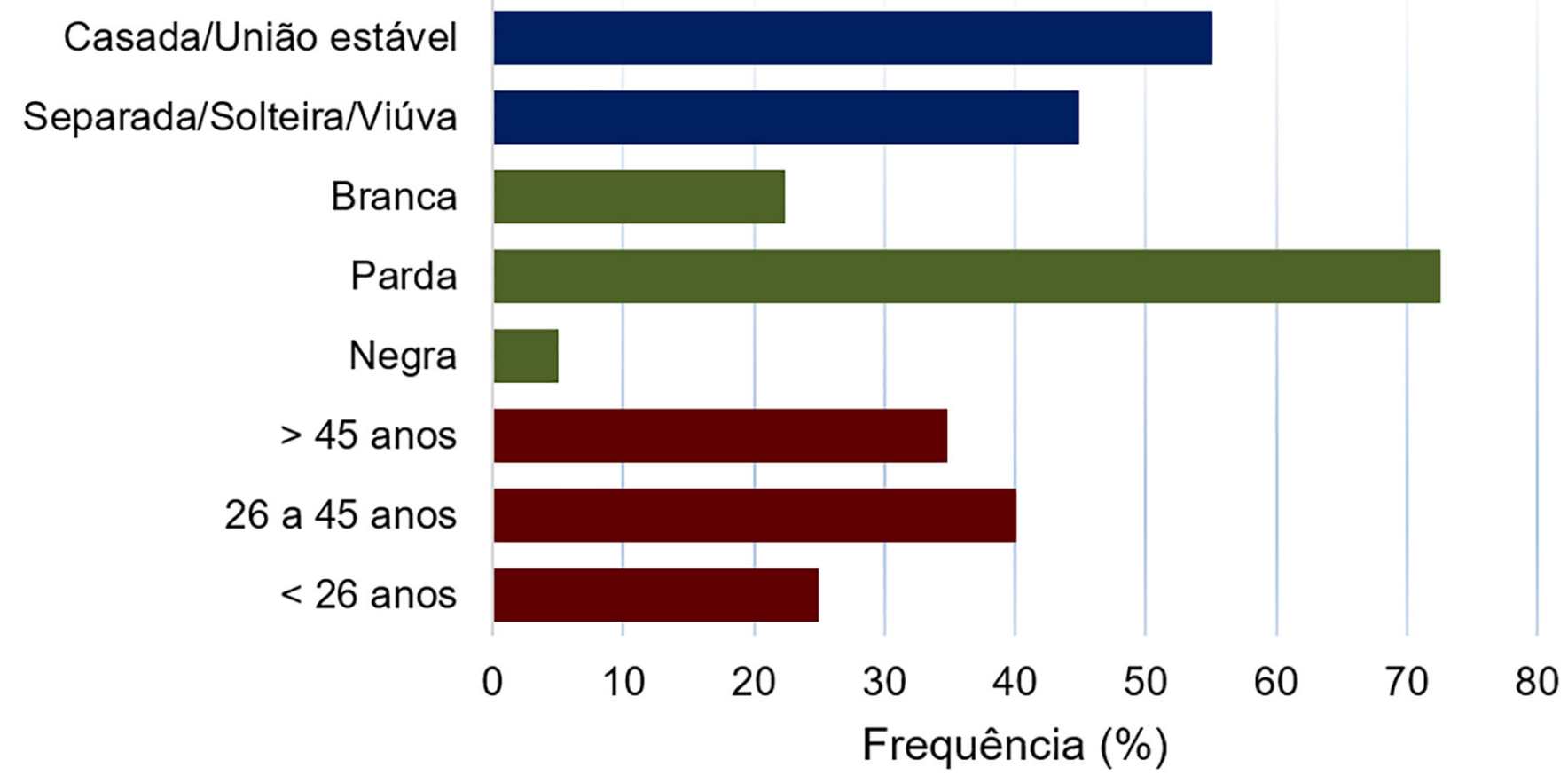

Gráfico 2. Comportamento sexual e reprodutivo das mulheres atendidas na policlínica de referência da UVV, no período de 2011 a 2016.
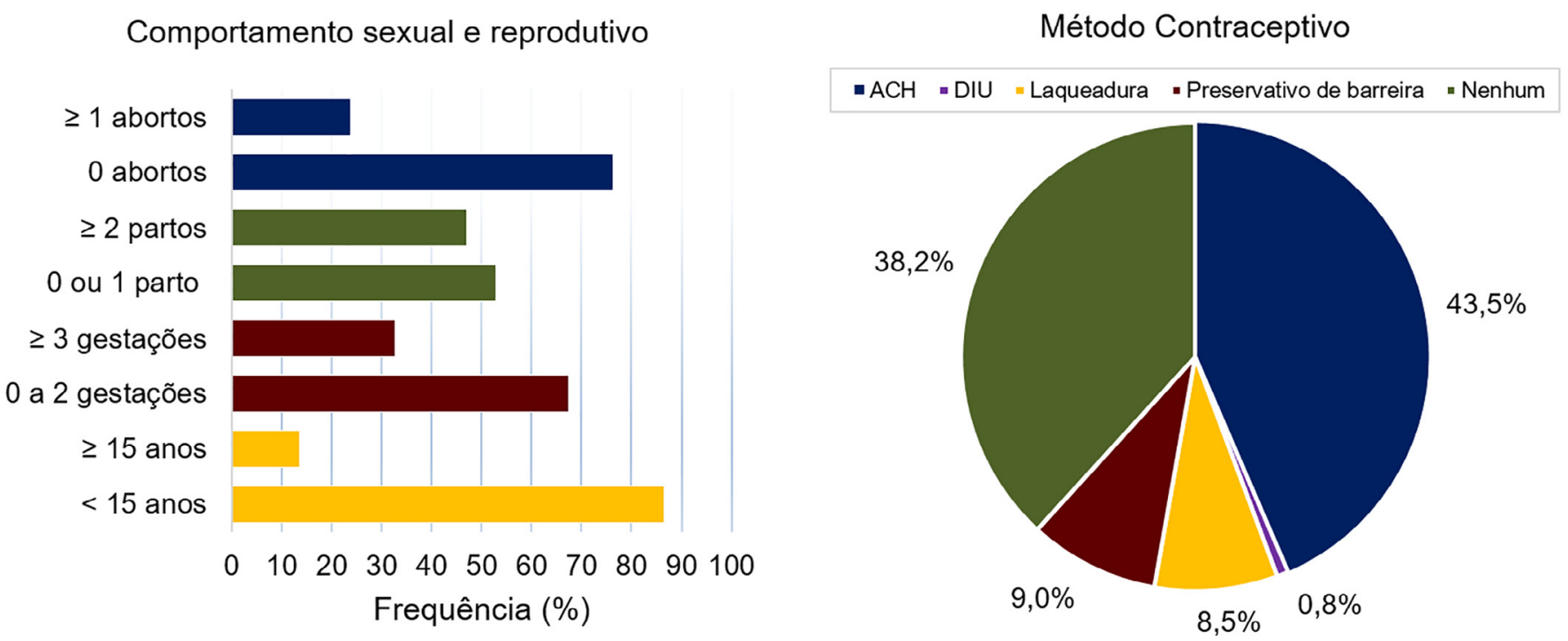

Legenda: ACH: Anticoncepcionais hormonais; DIU: Dispositivo intrauterino.

Nesse espaço amostral, $184(31,2 \%)$ das pacientes realizaram o exame citopatológico, e a partir dele, apenas 20 mulheres apresentaram resultados indicativos de colposcopia, sendo 13 delas com idade igual ou superior a 30 anos (Tabela 1). 
Tabela 1. Exame de colposcopia conforme a idade da paciente.

\begin{tabular}{|c|c|c|c|}
\hline COLPOSCOPIA & Indicado & Não indicado & \\
\hline$<30$ anos & $7(3,8 \%)$ & $37(20,1 \%)$ & \\
\hline$\geq 30$ anos & $13(7,1 \%)$ & $127(69 \%)$ & \\
\hline Total & 20 & 164 & 184 \\
\hline
\end{tabular}

Observamos que as maiores prevalências de achados colposcópicos sugestivos de maior gravidade foram encontradas em mulheres com 30 ou mais anos, dados esses confirmados pela biópsia, considerada padrão ouro no diagnóstico das lesões precursoras de câncer cervical (Gráfico 3).

Gráfico 3. Características das pacientes e os resultados do preventivo e biópsia.

\section{Exame Citopatológico}

7

6

5

4

3

2

1

0

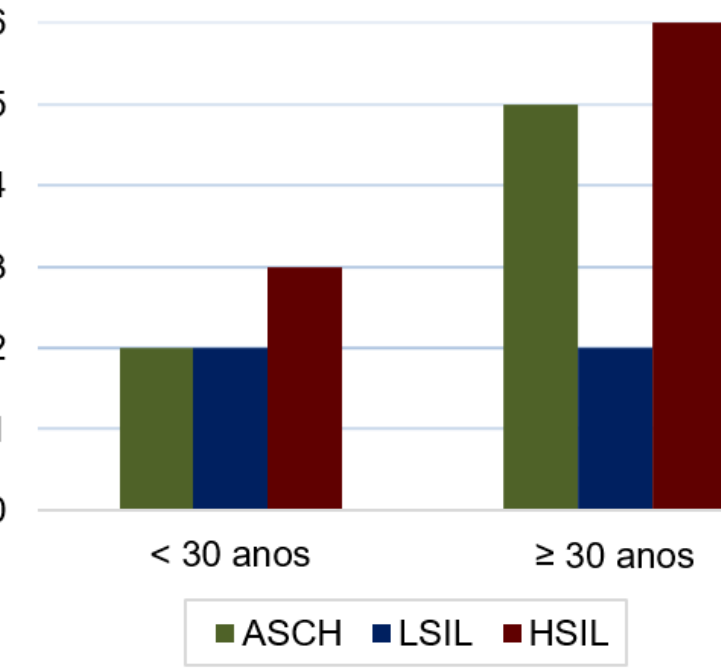

Resultado de biópsia

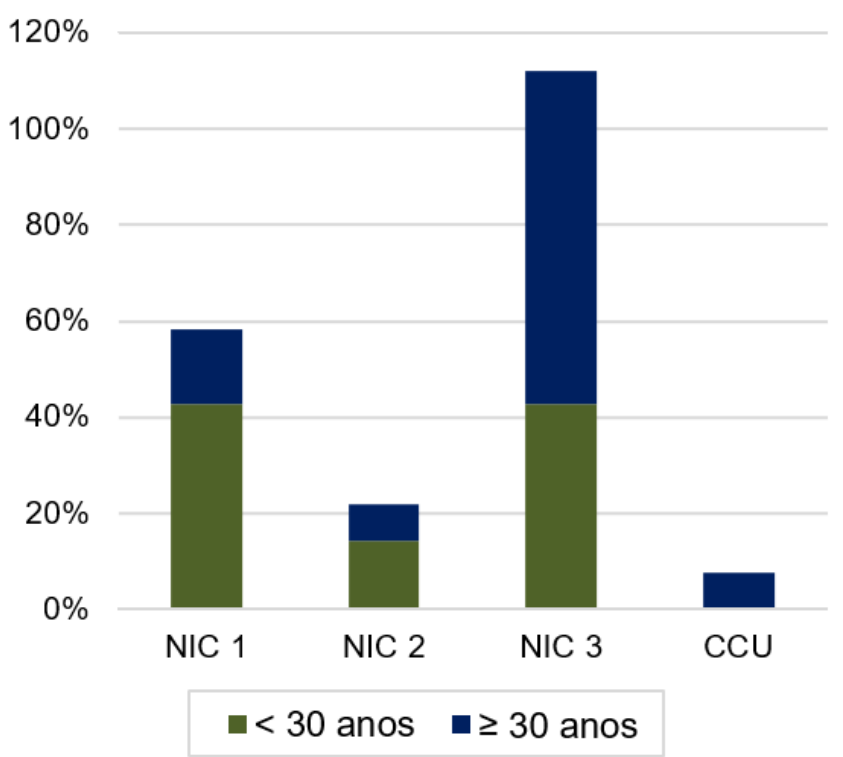

Legenda: SCH: Células escamosas atípicas; LSIL: Lesão intraepitelial escamosa de baixo grau; HSIL: Lesão intraepitelial escamosa de alto grau; NIC: Neoplasia intraepitelial cervical; CCU: Câncer do colo de útero.

Em relação aos riscos associados, mulheres com 3 partos ou mais tem 1,68 mais chances de desenvolver carcinoma in situ (NIC 3) ou carcinoma invasivo comparado às mulheres com menos de 3 partos. Analogamente, identificou-se que mulheres solteiras apresentam 4,4 mais chances de desenvolver essas mesmas lesões quando comparadas às mulheres casadas. Além disso, pacientes em uso de $\mathrm{ACH}$ apresentam 2,25 mais chances em desenvolver CCU em relação às demais que não faziam uso dessa contracepção (Tabela 2).

\section{DISCUSSÃO}

Em vista aos 590 prontuários analisados, 184 pacientes $(31,2 \%)$ procuraram o serviço da policlínica para realizar o exame citopatológico. Neste grupo, apenas $10,8 \%$ das pacientes realizaram a colposcopia para o seguimento do rastreio do CCU, sendo $65 \%$ delas com idade maior ou igual a 30 anos.

A cobertura para o rastreio do CCU realizado pelo serviço de atenção integral à saúde da mulher da policlínica da UVV, não atingiu a meta estipulada pelo Ministério da Saúde, ou seja, pelo menos $70 \%$ das 
Tabela 2. Resultados da biópsia e os riscos associados.

\begin{tabular}{|c|c|c|c|}
\hline \multicolumn{4}{|c|}{ NIC 3 ou carcinoma epidermóide } \\
\hline Partos & Sim & Não & Total \\
\hline$\geq 3$ partos & $9(\mathrm{OR} 1,68)$ & 4 & 13 \\
\hline$<3$ partos & 4 & 3 & 7 \\
\hline Total & 13 & 7 & \\
\hline Estado civil & Sim & Não & Total \\
\hline Solteira & $10($ OR 4,44$)$ & 3 & 13 \\
\hline Uso de $\mathrm{ACH}$ & Sim & Não & Total \\
\hline Sim & $3(\mathrm{OR} 2,25)$ & 6 & 9 \\
\hline Não & 2 & 9 & 11 \\
\hline Total & 5 & 15 & \\
\hline
\end{tabular}

Legenda: NIC: Neoplasia intraepitelial cervical; ACH: Anticoncepcionais hormonais.

mulheres atendidas. Mesmo diante de campanhas nacionais de conscientização para alertar as mulheres e a sociedade sobre a importância da prevenção e do diagnóstico precoce do câncer de mama e do CCU, a baixa cobertura do exame citopatológico infere em altas taxas de mortalidade feminina devido ao CCU, sendo a maioria desses casos detectado em estágio avançado. ${ }^{8}$

Nesse estudo, a associação estatística da variável idade não evidenciou risco de CCU. Embora não seja possível descartá-la, uma vez que a incidência de CCU atinge seu pico na quarta ou quinta década de vida da mulher, ${ }^{2}$ provavelmente explicado pela persistência ou reativação de infecções previamente adquiridas. ${ }^{9}$ Vale considerar que adultos jovens sexualmente ativos, principalmente no início da vida sexual, estão mais expostos ao risco de adquirir o HPV, ${ }^{4,10}$ ainda que as infecções e as lesões de baixo grau, em regra, regredirão espontaneamente antes dos 25 anos de idade. ${ }^{11}$

Ao analisar os dados colpocitológico e biópsia, observou-se uma prevalência de 1,52\% das pacientes com um resultado de lesão intraepitelial de alto grau (HSIL), valor 11 vezes maior que o resultado obtido num estudo equivalente, realizado pelo Centro Universitário de Anápolis - UniEvangélica - Anápolis/GO. ${ }^{12}$ Assim como foi encontrado um valor 3,8 vezes maior em relação à taxa esperada pelo Ministério da Saúde (superior a 0,4\%). ${ }^{12}$

Para relação entre lesão do colo do útero e idade da paciente, o presente estudo adotou a idade de 30 anos, ${ }^{2}$ uma vez que a partir dessa idade as lesões por HPV podem ser persistentes. ${ }^{11}$ Dentre as 7 pacientes analisadas com diagnóstico histopatológico de NIC 1, lesão de comportamento benigno e de regressão espontânea, apenas 2 incluíam a faixa etária de 35 a 49 anos. Este período corresponde ao pico de incidência das lesões precursoras e antecede o pico de mortalidade pelo câncer de colo de útero. ${ }^{13}$ Sob esta mesma análise, dentre as 6 pacientes com diagnóstico histopatológico de NIC 2, NIC 3 e CCU (especificamente carcinoma epidermóide), 5 tinham idade maior que 35 anos, sendo aquela diagnosticada com carcinoma epidermóide com 36 anos. Estudos recentes ${ }^{14,15}$ detectaram que a faixa etária prevalente de lesão precursora de CCU compreende idades de 26 a 58 anos. O presente estudo mostrou semelhança, com abrangência da faixa etária de 18 a 60 anos.

Em relação aos riscos, observou-se uma associação direta entre o número de gestações e o risco de CCU. 1,15,16 Mulheres com 3 partos ou mais tem 1,68 mais chances de desenvolver NIC 3 ou carcinoma invasivo, comparado às mulheres com menos de 3 partos. Esses resultados refletem a baixa adoção de métodos contraceptivos de barreira, ${ }^{1} \mathrm{e}$ uma maior possibilidade no número de gestações expondo a mulher à situação de risco ao CCU. ${ }^{16}$ 
Analogamente, identificou-se que mulheres solteiras apresentam 4,4 mais chances de desenvolver essas mesmas lesões quando comparadas às mulheres casadas. Analisando o fator tempo de relacionamento monogâmico, há um consenso de que mulheres em relações estáveis estão menos susceptíveis ao CCU.1,9 E não diferente no presente trabalho, constatou-se que mulheres em relacionamentos curtos ou solteiras que mantém vida sexual ativa, apresentaram 4,4 mais chances de desenvolver carcinoma in situ ou CA invasor comparado às mulheres em relacionamentos duradouros ou casadas. ${ }^{1,10}$

Por isso são importantes campanhas com orientações direcionadas às pacientes e seus parceiros(as), com o objetivo de reduzir a transmissão do HPV. Os envolvidos devem ser informados de que podem ser infecciosos, mesmo na ausência de lesões visíveis.

Pesquisas sugerem que anticoncepcional hormonal $(\mathrm{ACH})$ exerce potencial efeito na carcinogênese cervical. ${ }^{10,11,17}$ No Brasil, estudos apontaram que $28,8 \%$ das mulheres com CCU faziam uso de ACH. ${ }^{1}$ Acreditase que com o uso de $\mathrm{ACH}$, mulheres passam a realizar atividade sexual desprotegida com mais frequência, tornando mais vulneráveis ao contato com o vírus. Nessa mesma perspectiva, o presente trabalho constatou que pacientes em uso de $\mathrm{ACH}$ apresentam 2,25 mais chances em desenvolver $\mathrm{CCU}$ em relação às demais.

Cabe destacar que ainda há pesquisas com associações divergentes quanto ao uso de ACH e risco de CCU. Estudo recente ${ }^{18}$ apontou que mulheres que usam $\mathrm{ACH}$ têm incidência mais baixa de infecção por HPV comparado às não usuárias, provavelmente explicado pela influência do estrógeno na resposta imunológica da produção de anticorpos, com aumento da expressão de lgA e lgG no muco cervical.

Fatores como comportamento sexual, ${ }^{10,19}$ tabagismo ${ }^{11}$ e iniciação sexual precoce, ${ }^{1}$ parecem influenciar os mecanismos que determinam a regressão ou a persistência da infecção, bem como a progressão para lesões precursoras ou câncer. ${ }^{1,6}$ Todavia, a grande maioria dos prontuários aqui analisados não continham tais dados, e dessa forma, impossibilitou correlacionar os fatores de risco à prevalência de CCU. Outra limitação, corresponde ao fato de não termos acesso à tipagem do DNA do HPV para identificar a forma latente dessa infecção, impossibilitando calcular a prevalência dos genótipos de HPV. ${ }^{20}$ Assim, nossa análise principal consiste no estudo da prevalência e fatores associados ao CCU.

\section{CONCLUSÃO}

No presente trabalho, observou-se uma alta prevalência de resultado de HSIL, maior, inclusive, do que o esperado pelo Ministério da Saúde. Quanto aos riscos associados analisados, destacam-se uso de $\mathrm{ACH}$, idade maior de 29 anos e mulheres solteiras. Os fatores de risco de CCU não se restringem apenas à idade e ao comportamento sexual das mulheres, mas também englobam tabagismo, paridade e diferentes genótipos do HPV. Destarte, essas informações são essenciais para o desenho de estratégias preventivas eficazes contra o CCU, e assim, o estudo reforça a importância da prevenção do câncer do colo do útero com a elaboração de programas de rastreamento organizados efetivos para a população.

\section{AGRADECIMENTOS}

A Universidade Vila Velha.

\section{Contribuição dos autores}

Concepção e/ou delineamento do estudo: ASSGM, IBO, FACV, JFR, ARMS. Aquisição, análise ou interpretação dos dados: ASSGM, IBO, FACV, JFR. 
Redação preliminar: ASSGM, IBO.

Revisão crítica da versão preliminar: ARMS, GECR, WSR.

Todos os autores aprovaram a versão final e concordaram com prestar contas sobre todos os aspectos do trabalho.

\section{Conflitos de interesse}

Eu, ARMS, autor responsável pelo manuscrito "Rastreio e associações ao câncer cervical", declaro que nenhum dos autores deste estudo possui qualquer tipo de interesse abaixo descrito, ou outros que configurem o chamado conflito de interesse. Declaro que o manuscrito apresentado não recebeu qualquer suporte financeiro da indústria ou de outra fonte comercial e nem eu, nem os demais autores ou qualquer parente em primeiro grau possuímos interesses financeiros/outros no assunto abordado no manuscrito. Em caso contrário, especifico, abaixo, qualquer associação que possa representar um conflito de interesse que eu e/ou os demais autores ou seus parentes de primeiro grau tive(mos) nos últimos cinco anos com empresas privadas e/ou organizações, mesmo sem fins lucrativos — por exemplo: participação em inventos/ desenvolvimento de software, aparelho, técnica de tratamento ou laboratorial, equipamentos, dispositivos ou tecnologias; participações e atividades de consultoria e/ou palestras; propriedade intelectual; participação acionária; situações de licenciamento de patentes etc.

\section{REFERÊNCIAS}

1. International Agency of Research on Cancer (IARC). ICO/IARC Information Centre on HPV and Cancer. Brazil human papillomavirus and related cancers, fact sheet [Internet]. Lyon: IARC; 2019; [acesso em ANO Jun 17]. Disponível em: https://hpvcentre.net/statistics/reports/BRA_FS.pdf

2. Instituto Nacional de Câncer José Alencar Gomes Da Silva (INCA). Parâmetros técnicos para o rastreamento do câncer do colo do útero [Internet]. Rio de Janeiro (RJ): INCA; 2019. Disponível em: https://www.inca.gov.br/sites/ufu.sti.inca.local/files//media/document// parametros_tecnicos_colo_do_utero_2019.pdf

3. Haverkos HW, Haverkos GP, O'Mara M. Co-carcinogenesis: human papillomaviruses, coal tar derivatives, and squamous cell cervical cancer. Front Microbiol. 2017 Out;8:2253. DOI: https://doi.org/10.3389/fmicb.2017.02253

4. Nakagawa JTT, Schirmer J, Barbieri M. Vírus HPV e câncer de colo de útero. Rev Bras Enferm. 2010;63(2):307-11.

5. Anttila A, Von Karsa L, Aasmaa A, Fender M, Patnick J, Rebolj M, et al. Cervical cancer screening policies and coverage in Europe. Eur J Cancer. 2009 Oct;45(15):2649-58. DOI: https://doi.org/10.1016/j.ejca.2009.07.020

6. Instituto Nacional de Câncer José Alencar Gomes Da Silva (INCA). Diretrizes brasileiras para o rastreamento do câncer do colo do útero [Internet]. Rio de Janeiro (RJ): INCA;2016. Disponível em: http://www1.inca.gov.br/estimativa/2018/estimativa-2018.pdf

7. Melo SCC, Prates L, Carvalho MDB, Marcon SS, Pelloso SM. Alterações citopatológicas e fatores de risco para a ocorrência do câncer de colo uterino. Rev Gaúcha Enferm Online. 2009 Dez;30(4):602-8. DOI: https://doi.org/10.1590/S1983-14472009000400004

8. Silva ML, Nunes JSS, Oliveira KS, Leite TAS. Conhecimento de mulheres sobre câncer de colo do útero: uma revisão integrativa. Braz J Health Rev. 2020;3(4):7263-75. DOI: https://doi.org/10.34119/bjhrv3n4-005

9. Palefsky JM, Hirsch MS, Bloom A. Human papillomavirus infections: epidemiology and disease associations [Internet]. Waltham:UpToDate; 2018. Disponível em: https://www.uptodate.com/contents/human-papillomavirus-infections-epidemiology-and-disease-associations

10. International Collaboration of Epidemiological Studies of Cervical Cancer. Cervical carcinoma and sexual behavior: collaborative reanalysis of individual data on 15,461 women with cervical carcinoma and 29,164 women without cervical carcinoma from 21 epidemiological studies. Cancer Epidemiol Biomark Prev. 2009 Apr;18(4):1060-9. DOI: https://doi.org/10.1158/1055-9965.EPI-08-1186

11. International Collaboration of Epidemiological Studies of Cervical Cancer. Cervical cancer and hormonal contraceptives: collaborative reanalysis of individual data for 16,573 women with cervical cancer and 35,509 women without cervical cancer from 24 epidemiological studies. Lancet. 2007 Nov;370(9599):1609-21. DOI: https://doi.org/10.1016/S0140-6736(07)61684-5 
12. Lemos ARM, Silva MS, Segati KD. Lesão de alto grau e carcinoma escamoso: um estudo de prevalências em pacientes atendidas pelo Sistema Único de Saúde (SUS) na cidade de Anápolis, GO, Brasil. RBAC. 2017;49(2):152-7. DOI: https://doi.org/10.21877/2448-3877.201600494

13. Alves JF, Oliveira WLS, Mendonça BOM, Oliveira VCC, Nogueira DS, Barros EJ, et al. Exame colpocitológico (papanicolau): O conhecimento das mulheres sobre o preventivo no combate do câncer de colo do útero. Rev Faculdade Montes Belos (FMB). 2016; [citado ANO Mês dia]; 9(2):125-41. Disponível em: http://revista.fmb.edu.br/index.php/fmb/article/view/228/205

14. Lima WF, Barbosa SLF, Oliveira GAL. Profile of gynecological cytopathology exams carried out in a north Piauí clinic: documental analysis. Res Soc Dev. 2020;9(7):e325974440. DOI: https://doi.org/10.33448/rsd-v9i7.4440

15. Silva RCG, Silva ACO, Peres AL, Oliveira SR. Perfil de mulheres com câncer de colo do útero atendidas para tratamento em centro de oncologia. Rev Bras Saude Mater Infant. 2018 Out/Dez;18(4):695-702. DOI: https://doi.org/10.1590/1806-93042018000400002

16. Muñoz N, Franceschi S, Bosetti C, Moreno V, Herrero R, Smith JS, et al. Role of parity and human papillomavirus in cervical cancer: the IARC multicentric case-control study. Lancet. 2002 Mar;359(9312):1093-101. DOI: https://doi.org/10.1016/S0140-6736(02)08151-5

17. Smith JS, Green J, Gonzalez AB, Appebly P, Peto J, Plummer M, et al. Cervical cancer and use of hormonal contraceptives: a systematic review. Lancet. 2003 Apr;361(9364):1159-67. DOI: https://doi.org/10.1016/s0140-6736(03)12949-2

18. Uchimura NS, Ribalta JCL, Focchi J, Baracat EC, Uchimura TT. Influência do uso de anticoncepcionais hormonais orais sobre o número de células de Langerhans em mulheres com captura híbrida negativa para papilomavírus humano. Rev Bras Ginecol Obstet. 2005 Dez;27(12):726-30. DOI: https://doi.org/10.1590/S0100-72032005001200004

19. Monteiro DLM, Trajano AJB, Silva KS, Russomano FB. Pre-invasive cervical disease and uterine cervical cancer in Brazilian adolescents: prevalence and related factors. Cad Saúde Pública. 2006 Dez;22(12):2439-48. DOI: https://doi.org/10.1590/S0102-311X2006001200004

20. Oliveira GR, Vieira VC, Barral MFM, Döwich V, Soares MA, Conçalves CV, et al. Fatores de risco e prevalência da infecção pelo HPV em pacientes de Unidades Básicas de Saúde e de um Hospital Universitário do Sul do Brasil. Rev Bras Ginecol Obstet. 2013 Mai;35(5):22632. DOI: https://doi.org/10.1590/S0100-72032013000500007 